\title{
Philosophiques
}

\section{Doyon et T. Breyer, Normativity in Perception, Palgrave MacMillan, 2015}

\section{Corrine Lajoie}

Volume 43, numéro 1, printemps 2016

URI : https://id.erudit.org/iderudit/1036477ar

DOI : https://doi.org/10.7202/1036477ar

Aller au sommaire du numéro

Éditeur(s)

Société de philosophie du Québec

ISSN

0316-2923 (imprimé)

1492-1391 (numérique)

Découvrir la revue

Citer ce compte rendu

Lajoie, C. (2016). Compte rendu de [M. Doyon et T. Breyer, Normativity in Perception, Palgrave MacMillan, 2015]. Philosophiques, 43(1), 159-162.

https://doi.org/10.7202/1036477ar

Ce document est protégé par la loi sur le droit d'auteur. L'utilisation des services d'Érudit (y compris la reproduction) est assujettie à sa politique d'utilisation que vous pouvez consulter en ligne.

https://apropos.erudit.org/fr/usagers/politique-dutilisation/ 


\section{Doyon et T. Breyer, Normativity in Perception, Palgrave MacMillan, 2015}

Si la tradition philosophique s'est plus largement intéressée à la dimension morale ou éthique de la normativité, dans l'introduction de l'ouvrage Normativity in Perception (2015), Maxime Doyon et Thiemo Breyer argumentent en faveur d'un élargissement du concept de norme, lequel concernerait l'agir en général. Nous discernons en effet plusieurs types de normes à l'œuvre dans notre expérience quotidienne: qu'on pense seulement à notre perméabilité aux normes sociales, légales, esthétiques, langagières, scientifiques et même académiques en vigueur dans un milieu ou une culture donnés. Le plus souvent, ces normes n'agissent pas isolément; elles communiquent dans l'expérience pour créer ce que les auteurs appellent « $a$ multi-faceted normative space that allows or encourages certain behaviors and practices and disallows and discourages others " (NP I). Les différentes normes que nous adoptons induisent des conséquences concrètes sur nos comportements, nos discours et nos attentes, mais elles influencent aussi plus holistiquement la nature même de notre relation au monde. Elles constituent en quelque sorte l'horizon normatif du milieu dans lequel nous vivons et sont la plupart du temps si bien intégrées qu'on se surprend lorsqu'un individu s'y soustrait ou marque un écart à leur endroit. Le cadre normatif qui oriente nos actions peut en outre servir à distinguer les comportements «normaux» des comportements dits «anormaux», et par conséquent le sujet «normal» du sujet «anormal» ou "pathologique».

Même si nous attribuons un rôle important au travail des normes dans plusieurs sphères de notre vie, il peut nous sembler plus contre-intuitif de parler de normativité dans le domaine de la perception, et le problème, à quelques exceptions près - citées en introduction par les éditeurs -, est relativement nouveau dans la littérature philosophique. L'enjeu de la normativité dans la perception soulève à juste titre plusieurs questions: d'abord, comment peut-on parler d'une bonne ou d'une mauvaise perception, et quand pouvons-nous dire qu'une perception est normativement adéquate, optimale ou réussie? Les normes en jeu seraient-elles a priori ou a posteriori? Impliquent-elles nécessairement un appareillage conceptuel ou peut-on les trouver sur un plan pré-conceptuel? Quelles conséquences (épistémologiques, ontologiques, métaphysiques) entraînent-elles pour une théorie de la perception? Enfin, comment le caractère normatif de nos pratiques perceptuelles s'arrime-t-il au contexte intersubjectif, historique et social dans lequel elles se déploient?

À la lumière de ces questions, l'objectif de l'ouvrage est double: faire travailler conjointement les intuitions d'auteurs d'horizons philosophiques distincts et aborder à nouveaux frais la question de la normativité en la situant au cœur d'une analyse de la perception. En ouvrant un dialogue trop souvent timide entre la tradition analytique et continentale en philosophie, 
les articles réunis abordent la question de la normativité dans la perception à travers quatre axes principaux. Les sections de l'ouvrage examinent respectivement: I) un survol de certains enjeux fondamentaux (Charles Siewert, Maxime Doyon, Michael Madary); 2) le statut particulier des illusions et des hallucinations (David Morris, Matthew Ratcliffe); 3) le rapport de la normativité perceptuelle avec l'horizon social et affectif du sujet (Shaun Gallagher, Maren Wehrle, Thiemo Breyer) ; 4) une analyse d'enjeux plus strictement épistémologiques (Aude Bandini, Arnaud Dewalque, Virginie Palette, Valérie Aucouturier).

Dans leur introduction, Doyon et Breyer présentent un survol des différentes inflexions qu'a pu adopter l'intérêt philosophique pour la question de la normativité, tant en philosophie de l'action qu'en philosophie de l'esprit ou du langage. Le plus souvent abordée par un biais épistémologique, une réflexion naissante et plus ou moins explicite sur le statut normatif de la perception émerge toutefois dans certains débats philosophiques au $\mathrm{xx}^{\mathrm{e}}$ siècle. Si la perception a l'ambition tacite de saisir le monde tel qu'il est, elle doit permettre la formulation de jugements épistémiques valides: une analyse épistémologique du problème s'intéresse à l'objectivité de notre rapport aux objets en vue de formuler des énoncés prédicatifs valides, soumis à la dialectique vrai-faux. Les éditeurs citent par ailleurs le débat entre les approches conceptuelles et non conceptuelles de la normativité dans la perception. Ainsi, par exemple, la tradition phénoménologique défend contre le modèle conceptualiste un seuil d'activité perceptive qui ne mobilise a priori aucune capacité rationnelle ou judicative. L'introduction, par Edmund Husserl, du rôle fondamental du corps dans la perception fournit des munitions importantes à l'approche non conceptualiste et traite de la question de la normativité dans la perception à partir d'une analyse phénoménologique de l'expérience d'un sujet incarné, situé et dynamique.

En raison du manque d'espace, nous n'entrerons pas dans le détail des douze articles qui composent l'ouvrage. Nous proposons plutôt de sélectionner et résumer certains arguments afin de former un bref panorama qui mette en saillance la pluralité des angles de lecture possibles.

Au chapitre I, Charles Siewert s'intéresse à l'apport normatif de l'expérience sensible (perceptive, plus précisément) dans la formulation de jugements sur une situation donnée. S'il est généralement admis que nous pouvons attribuer une valeur normative à nos jugements, puisque nous les considérons comme étant vrais ou faux, et légitimes ou illégitimes en vertu de critères normatifs vérifiables, l'analyse du caractère normatif de l'expérience sensible est plus ambiguë. En effet, comment l'expérience subjective d'un état de choses perçu (le "what-is-it-likeness of experience " des phénoménologues) peut-elle être dite normative et, en outre, de quelle manière confèret-elle un statut normatif aux jugements qu'elle permet de fonder? La question est d'intérêt puisqu'elle contribue à établir une théorie de la conscience qui rende compte du travail collaboratif de l'expérience sensible 
(ou de notre corporéité, au sens large), et des processus cognitifs dans la perception. À cet égard, l'article de Siewert est situé en tête d'ouvrage de manière particulièrement intéressante: sa réflexion se situe à la charnière de questionnements qui seront repris au fil du recueil.

Pour Siewert, plusieurs exemples fournissent la preuve d'une tendance naturelle à ajuster notre perception (en nous approchant, en nous éloignant, en inclinant la tête, en balayant l'espace du regard, etc.), lorsque nous devons réaliser une tâche précise ou nous orienter dans l'espace. La majorité de nos activités quotidiennes (chercher nos clefs, conduire une voiture, se servir un verre d'eau) sont réalisées sans l'apport de processus cognitifs complexes. Pour l'auteur, cette familiarité pré-réflexive avec l'espace doit pouvoir alimenter une réflexion sur le rôle de l'expérience sensible dans l'articulation de jugements normatifs. En somme, je suis généralement mieux outillé pour formuler un jugement sur un objet perçu, à proportion de la précision et de la distinction avec lesquelles je le perçois. Siewert propose l'hypothèse d'une «constance phénoménale» pour expliquer l'invariance de certaines propriétés à travers le flux perceptuel de l'expérience. Cette constance phénoménale travaille de concert avec le dévoilement progressif de nouveaux profils et forme un noyau de variations relatif à l'activité dynamique (ou exploratoire) du sujet. De manière générale, l'argument de Siewert s'appuie sur l'idée que l'expérience perceptive peut être enrichie et ménager ainsi un accès qualitativement meilleur à l'objet perçu, lequel accès valide le plus souvent la valeur de vérité des jugements normatifs qu'il permet de fonder.

$\mathrm{Au}$ chapitre II, Maxime Doyon défend la supériorité du modèle phénoménologique (husserlien et merleau-pontien) dans une analyse du caractère normatif de la perception, à partir d'une description du rôle central qu'y joue l'autoréférentialité. La stratégie de l'auteur consiste à mettre dos à dos deux approches concurrentes du caractère normatif de la perception à partir d'un terrain qui leur est commun : celui d'une analyse de l'articulation entre conscience de soi ( «self-consciousness") et conscience du monde extérieur ("perceptual consciousness») au sein de nos pratiques perceptuelles. S'il est vrai que la tradition kantienne dans laquelle s'inscrit John McDowell et la tradition phénoménologique inaugurée par Edmund Husserl s'entendent sur un facteur essentiel d'autoréférentialité dans la perception, il y a néanmoins désaccord sur la nature de son lien avec notre ouverture au monde. McDowell explique la normativité dans la perception comme une capacité (rationnelle et langagière) d'attester réflexivement de nos expériences et de notre situation, tandis que les analyses phénoménologiques de l'intentionnalité motrice chez Husserl et Merleau-Ponty argumentent en faveur d'une structure normative liminaire, située à un niveau pré-réflexif et directement liée à la contribution fondamentale du corps (et de son système sensori-moteur) dans la perception.

Au chapitre IV, David Morris rejette la compréhension de l'illusion perceptive comme erreur et s'intéresse au caractère vécu d'une incompatibi- 
lité ou d'une contradiction entre un bagage de normes perceptives exogènes et l'émergence de normes endogènes ponctuelles et coalescentes au déploiement de l'expérience du sujet. Pour Morris, dont l'article se situe dans le sillage des analyses de Maurice Merleau-Ponty, les illusions ne nous donnent pas un monde "faux" ou "erroné", mais elles rompent plutôt avec son style habituel et organisent les éléments du champ perceptif autour d'un nouveau sens. L'approche phénoménologique de l'auteur s'appuie sur des cas classiques d'illusions et sur des intuitions empruntées à la recherche en biologie de l'évolution pour argumenter en faveur d'une structure normative plastique qui fait droit au caractère largement dynamique, temporel et adaptatif de nos pratiques perceptuelles. Avec ce modèle, l'auteur cherche à invalider l'idée d'une norme statique qui puisse s'appliquer indistinctement à l'ensemble de nos perceptions. Les normes perceptives émergent plutôt au sein de l'expérience, dans l'épaisseur et la densité d'un présent qui combine reprise, évolution, acquisition et habitudes.

Au chapitre VI, Maren Wehrle analyse l'entrelacement de la structure normative dans la perception avec les critères de "normalité » et d' "anormalité " qui en découlent. En s'intéressant particulièrement au concept de passivité chez Husserl et à sa thématisation d'un optimum perceptuel relatif à l'intérêt, Wehrle analyse l'émergence de critères normatifs incarnés, définis à différents niveaux par l'horizon d'attente, la «sensibilité normative » et le passé expérientiel du sujet perceptif. Si plusieurs auteurs (Butler, Foucault) ont largement analysé l'influence des normes sociales sur notre corps, Wehrle insiste sur une structure normative primordiale, située même en deçà de l'influence des constellations de pouvoir discursives, politiques ou technologiques. À un niveau plus élémentaire, la phénoménologie s'intéresse notamment aux conséquences normatives des processus pré-réflexifs de sédimentation et d'habituation à l'œuvre dans la perception.

Dans l'ensemble, Normativity in Perception propose un dialogue fécond entre une diversité de travaux, et l'ouvrage signe une avancée pertinente pour la recherche en philosophie de la perception dans les domaines analytiques et continentaux. Il y a fort à parier qu'il pourra aussi contribuer plus largement aux travaux consacrés à la question de la normativité dans divers champs de recherche en sciences humaines. 